\title{
The risk of fall-related hospitalisations at entry into permanent residential aged care
}

\author{
Maria C. Inacio ${ }^{1,2^{*}}$, Max Moldovan ${ }^{1}$, Craig Whitehead ${ }^{3,4}$, Janet K. Sluggett ${ }^{1,2,5}$, Maria Crotty ${ }^{3,4}$, Megan Corlis ${ }^{6}$,
} Renuka Visvanathan ${ }^{7,8,9}$, Steve Wesselingh ${ }^{1}$ and Gillian E. Caughey ${ }^{1,2,10}$

\begin{abstract}
Background: Entering permanent residential aged care (PRAC) is a vulnerable time for individuals. While falls risk assessment tools exist, these have not leveraged routinely collected and integrated information from the Australian aged and health care sectors. Our study examined individual, system, medication, and health care related factors at PRAC entry that are predictors of fall-related hospitalisations and developed a risk assessment tool using integrated aged and health care data.
\end{abstract}

Methods: A retrospective cohort study was conducted on $N=32,316$ individuals $\geq 65$ years old who entered a PRAC facility (01/01/2009-31/12/2016). Fall-related hospitalisations within 90 or 365 days were the outcomes of interest. Individual, system, medication, and health care-related factors were examined as predictors. Risk prediction models were developed using elastic nets penalised regression and Fine and Gray models. Area under the receiver operating characteristics curve (AUC) assessed model discrimination.

Results: $64.2 \%$ ( $N=20,757)$ of the cohort were women and the median age was 85 years old (interquartile range 80-89). After PRAC entry, 3.7\% ( $N=1209)$ had a fall-related hospitalisation within 90 days and $9.8 \%(N=3156)$ within 365 days. Twenty variables contributed to fall-related hospitalisation prediction within 90 days and the strongest predictors included fracture history (sub-distribution hazard ratio $(\mathrm{sHR})=1.87,95 \%$ confidence interval (Cl) 1.63-2.15), falls history ( $\mathrm{sHR}=1.41,95 \% \mathrm{Cl} 1.21-2.15)$, and dementia ( $\mathrm{SHR}=1.39,95 \% \mathrm{Cl} 1.22-1.57)$. Twenty-seven predictors of fallrelated hospitalisation within 365 days were identified, the strongest predictors included dementia $(\mathrm{sHR}=1.36,95 \% \mathrm{Cl}$ 1.24-1.50), history of falls ( $\mathrm{sHR}=1.30,95 \% \mathrm{Cl} 1.20-1.42)$ and fractures $(\mathrm{sHR}=1.28,95 \% \mathrm{Cl} 1.15-1.41)$. The risk prediction models had an AUC of 0.71 (95\%Cl 0.68-0.74) for fall-related hospitalisations within 90 days and 0.64 (95\%Cl 0.62-0.67) for within 365 days.

Conclusion: Routinely collected aged and health care data, when integrated at a clear point of action such as entry into PRAC, can identify residents at risk of fall-related hospitalisations, providing an opportunity for better targeting risk mitigation strategies.

Keywords: Falls, Injury, Aged care, Risk-prediction

\footnotetext{
${ }^{*}$ Correspondence: maria.inacio@sahmri.com

${ }^{1}$ Registry of Senior Australians, South Australian Health and Medical

Research Institute, PO Box 11060, Adelaide, SA 5001, Australia

Full list of author information is available at the end of the article
}

\begin{abstract}
Background
Globally, over 37 million falls require medical attention each year, with at-least 165,000 injury related hospitalisations due to falls occurring in Australia alone [1-3]. While falls can be prevented, they continue to be a leading cause of reduced quality of life, need for aged care services, hospitalised injury, and injury-related deaths
\end{abstract}


for older people [4-7]. Health care costs from fall-related injuries in Australia in 2015-16 were estimated to be $\$ 3.6$ billion, $41 \%$ of all spending on injuries [8].

Individuals living in permanent residential aged care (PRAC) facilities (nursing homes or long-term care facilities) are most vulnerable to falls given their age, high burden of frailty, high prevalence of dementia, and use of psychotropic medications and medications that can cause orthostatic hypotension, among other contributing factors $[9,10]$. A prospective cohort study in six Australian PRAC facilities reported that $27 \%$ of hospitalisations over 12-months were fall injury related [11]. In order to implement evidence-based interventions for falls prevention in PRAC settings, adequate risk assessment, which incorporates identifying high risk individuals and potentially modifiable risk factors, must be employed [12]. While several risk assessment tools or prognostic models for falls risk identification have been developed for use in hospitals, community, or PRAC facilities [13-15], these tools have not leveraged the comprehensive information routinely collected in assessments and administrative records from the Australian aged care and health care sectors. Additionally, these tools have not examined the risk profile of individuals at key periods in their aged care journey, including first entry into PRAC. This may be a particularly vulnerable period in terms of falls risk, with residents unfamiliar with their new surroundings, together with considerable changes to medication regimen and care [16].

In 2017, the Registry of Senior Australians (ROSA) established the integration of the aged care and health care sectors' information for older Australians who have accessed aged care services, so the experience of individuals navigating these sectors can be understood [17]. The ROSA contains a population-based cohort of people using aged care services ( 2.9 million individuals) and has developed internationally agreed upon indicators of quality and safety for aged care settings, which includes a fallrelated hospitalisation indicator [18]. An examination of the 2016 cohort in residential aged care in Australia showed that $10.1 \%$ of South Australian residents had at least one fall that required hospitalisation or emergency department (ED) presentation and 3.3\% of facilities had higher than expected incidence of fall-related hospitalisations that year [18].

Using the comprehensive information that comprises ROSA our study aimed to: (1) examine individual, system, medication, and health care related factors at PRAC entry that are predictors of fall-related hospitalisations; (2) develop a fall-related hospitalisation risk assessment tool using integrated Australian aged care and health care data; (3) compare the newly developed fall-related hospitalisations risk assessment tool's performance to an existing falls-risk tool (i.e. Fracture Risk Assessment Tool for Community Dwelling older People (FRAT-up)) [19].

\section{Methods \\ Study design, setting, data source}

A retrospective cohort study was conducted using the ROSA [17]. This national dataset contains information on all individuals who have undergone an aged care eligibility assessment and accessed services that require this approval, namely permanent residential aged care, home care packages, transition care, and respite care. ROSA has established the linkage of the national aged care datasets from the Australian Institute of Health and Welfare (AIHW) National Aged Care Data Clearinghouse (NACDC), to the health care datasets from the Australian Government, including the Medicare Benefits Schedule (MBS) and Pharmaceutical Benefits Scheme (PBS), and state health authorities' hospitalisation data collections.

The specific datasets used in this study from the NACDC include the Aged Care Assessment Program (ACAP), Aged Care Funding Instrument (ACFI), episodes of Residential Aged Care Services, and National Death Index (NDI) [17]. The ACAP dataset provides information on the assessor, person seeking services, and recommended services at the time of eligibiligy assessment [20]. The ACFI dataset provides information on needs assessment performed at entry into PRAC. The episodes of Residential Aged Care Services dataset provides the services and dates they were received. The NDI dataset provides date and cause of death, coded using the International Statistical Classification of Diseases and Related Health Problems, Tenth Revision (ICD-10). The MBS dataset provides information on Australian Government subsidised health care services. The PBS dataset provides information on medications dispensed under the PBS (Australia's national prescription subsidy scheme), coded using the World Health Organisation Anatomical, Therapeutic and Chemical (ATC) classification [21]. The admitted patient datasets provide inpatient hospital encounters and the ED datasets include ED presentations, both are coded (or mapped) using ICD-10 Australian Modification (ICD-10-AM).

\section{Study cohort}

The study cohort included non-Indigenous individuals $\geq 65$ years old who entered PRAC in a South Australian facility between $01 / 01 / 2009$ and $31 / 12 / 2016$, who were not Department of Veterans' Affairs concession card holders $(N=32,316)$. 


\section{Outcome of interest}

Time to the first fall-related hospitalisation, defined as the first fall that resulted in hospitalisation or ED presentation, within 90 days or 365 days after entry into PRAC, were the outcomes of interest. Falls-related hospitalisations were determined using the 'external cause' code of the admitted data collection dataset for public hospitals and the 'diagnosis code' of the ED datasets using the ICD-10-AM codes listed on Supplementary Table 1. Falls with onset in hospital were not included. To ensure the cohort had a minimum follow-up of 365 days, the followup period was $01 / 01 / 2009$ to $31 / 12 / 2017$.

\section{Potential predictors of interest}

Individual factors (Table 1, Supplementary Tables 2 and 3 ) were determined at the aged care eligibility or entry into PRAC assessments and included: age, sex, preferred language (English vs. other), partner/marital status, Socio-economic Indexes for Areas' (SEIFA) relative socio-economic disadvantage index, SEIFA education and occupation index, and SEIFA economic resources index [22], weighted frailty index score [23], health conditions, functional limitations, and needs assessment regarding activities of daily living, behaviour, and complex health care needs at entry into care [24]. SEIFA 2016 was applied by matching the PRAC facility postcode to the SEIFA postal areas. Health conditions were ascertained from the aged care eligibligy assessment and entry into care assessment, where they are coded using the same four digit coding scheme [25]. For certain conditions, including diabetes, cerebrovascular disease, cardiovascular disease, and cancer, several conditions that may have fallen under that group (e.g. various types of cancer) were combined into one group. For the condition of dementia both assessments and the medication-based comorbidity index Rx-Risk-V indicator for dementia, which is based on medications used for treatment of dementia or its symptoms, in the 6 months prior to PRAC entry, were used [26].

Medication-related factors (Table 1, Supplementary Table 4) were ascertained from dispensing records in the 90-day period prior to entry into PRAC and included: number of unique medications dispensed (chemical substance ATC 5th level), sedative load rating (i.e. cumulative effect of medications with sedative properties) [27], and medication classes at the chemical subgroup ATC 4th level [21].

System and facility related factors (Table 1, Supplementary Table 2), were ascertained at the time of entry into PRAC and included: provider type (not-for-profit, for-profit, or government) and facility remoteness (based on the Accessibility/Remoteness Index of Australia [28], classified as major cities, inner regional, outer regional, or remote/very remote).

Health care-related factors (Table 1, Supplementary Table 5) were ascertained using the history of hospitalisations in the year prior to entry into PRAC and included: number of hospitalisations (unplanned and potentially preventable hospitalisations [29]), ED presentations (any and potentially preventable ED presentations [29]), and cumulative length of hospital stays. Additional health care-related factors were ascertained using the MBS subsidised health encounters (Table 1, Supplementary Table 6) in the year prior to PRAC entry and included: primary care or specialist attendances (geriatrician, palliative and pain), health assessments, team care arrangements, general practitioner (GP) management plan, and comprehensive medication review.

\section{Analysis}

The cohort and crude outcomes were described using means, standard deviation, medians, interquartile ranges (IQR), frequency and proportions. The study cohort was randomly split into five groups; four $(80 \%, N=25,853)$ were used for training the models and one (20\%, $N=6463$ ) was used for testing. An elastic nets penalised regression approach, using a Fine and Gray model with death as a competing risk, was used to select best subsets of variables predicting fall-related hospitalisations. The variables for age and sex (base model) were not penalised, and the year of cohort entry was added when fitting the final models. The predictor variables were selected from consensus among at-least three of four groups that penalised regressions were applied to the cross validation folds of the training samples, and assessed for the top 30 and 40 predictors of 90 days and 365 days falls-related hospitalisation incidence, respectively. To enhance model interpretability and avoid omitted-variable bias, if a single level of a multi-level categorical variable entered the model then the rest of levels were included (excluding the reference).

The proportional hazards assumption was tested using Schoenfeld residuals and its violation assessed from plotting log-log survival with respect to alternative quantiles of linear predictors. Subdistribution hazard ratios (sHR) and 95\% confidence intervals (CI) were presented. Each model's calibration was examined by bootstrapping bias-corrected estimates of predicted vs. observed values based on sub-setting predictions into intervals using 'calibrate() function' from $\mathrm{R}$ rms package. All calculations used complete-case analysis with $<4.7 \%$ of cases excluded due to missing data.

Model discrimination was examined using area under the receiver operating characteritics curve (AUC) [30] from the testing group and an additional out-of-sample 
Table 1 Highlights of individual and clinical characteristics at entry into permanent residential aged care, by fall-related hospitalisations within 90 days or 365 days of entry

\begin{tabular}{|c|c|c|c|c|c|}
\hline Characteristics & Total & Fall within 90 days & No fall within 90 days & Fall within 365 days & No fall within 365 days \\
\hline Total, N(\%) & 32,316 & 1209(3.7) & $31,107(96.3)$ & $3156(9.8)$ & $29,160(90.2)$ \\
\hline \multirow[t]{2}{*}{ Median Age (IQR), years } & 85(80-89) & $85(81-89)$ & 85(80-89) & 85(81-89) & 84(79-89) \\
\hline & $\mathrm{N}(\%)$ & $N(\%)$ & $\mathrm{N}(\%)$ & $N(\%)$ & $\mathrm{N}(\%)$ \\
\hline \multicolumn{6}{|l|}{ Sex } \\
\hline Women & $20,757(64.2)$ & $745(3.6)$ & $20,012(96.4)$ & 2083(10.0) & $18,674(90.0)$ \\
\hline Men & $11,559(35.8)$ & $464(4.0)$ & $11,095(96.0)$ & 1073(9.3) & $10,486(90.7)$ \\
\hline \multicolumn{6}{|l|}{ Facility Remoteness (ARIA) } \\
\hline Major cities & $24,799(76.7)$ & $957(3.9)$ & $23,842(96.1)$ & $2514(10.1)$ & $22,285(89.9)$ \\
\hline Inner regional & 4057(12.6) & $117(2.9)$ & $3940(97.1)$ & $321(7.9)$ & 3736(92.1) \\
\hline Outer regional & $3120(9.7)$ & 118(3.8) & $3002(96.2)$ & $290(9.3)$ & 2830(90.7) \\
\hline Remote or very remote & $340(1.1)$ & $17(5.0)$ & $323(95.0)$ & $31(9.1)$ & 309(90.9) \\
\hline \multicolumn{6}{|l|}{ Health Conditions ${ }^{b}$} \\
\hline Dementia & $16,334(50.5)$ & $734(4.5)$ & $15,600(95.5)$ & 1898(11.6) & $14,436(88.4)$ \\
\hline Delirium & $1286(4.0)$ & $81(6.3)$ & 1205(93.7) & 166(12.9) & 1120(87.1) \\
\hline History of fall & $6938(21.5)$ & $360(5.2)$ & 6578(94.8) & $867(12.5)$ & $6071(87.5)$ \\
\hline History of fracture & $5244(16.2)$ & $335(6.4)$ & 4909(93.6) & $667(12.7)$ & 4577(87.3) \\
\hline Osteoporosis & $6546(20.3)$ & $270(4.1)$ & 6276(95.9) & $740(11.3)$ & $5806(88.7)$ \\
\hline \multicolumn{6}{|c|}{ Rx-Risk-V Co-morbidity Category ${ }^{a}$} \\
\hline $0-1$ & 1875(5.8) & $47(2.5)$ & $1828(97.5)$ & 152(8.1) & 1723(91.9) \\
\hline $2-3$ & $5952(18.4)$ & 182(3.1) & $5770(96.9)$ & $529(8.9)$ & $5423(91.1)$ \\
\hline $4-5$ & $8555(26.5)$ & $314(3.7)$ & $8241(96.3)$ & $864(10.1)$ & $7691(89.9)$ \\
\hline $6-8$ & $10,825(33.5)$ & $448(4.1)$ & $10,377(95.9)$ & 1086(10.0) & 9739(90.0) \\
\hline $9+$ & $4627(14.3)$ & $210(4.5)$ & $4417(95.5)$ & $494(10.7)$ & 4133(89.3) \\
\hline \multicolumn{6}{|l|}{ ROSA Frailty Index Score ${ }^{a}$} \\
\hline 0.00 to 0.09 (least frail) & 1086(3.4) & $37(3.4)$ & 1049(96.6) & $96(8.8)$ & $990(91.2)$ \\
\hline 0.10 to 0.19 & $8550(26.5)$ & $273(3.2)$ & $8277(96.8)$ & $764(8.9)$ & 7786(91.1) \\
\hline 0.20 to 0.29 & $18,173(56.2)$ & 715(3.9) & $17,458(96.1)$ & 1834(10.1) & $16,339(89.9)$ \\
\hline 0.3 and over & $4442(13.7)$ & 183(4.1) & 4259(95.9) & $458(10.3)$ & 3984(89.7) \\
\hline \multicolumn{6}{|l|}{ Number of Unique Medications } \\
\hline $0-4$ & $3661(11.3)$ & $98(2.7)$ & 3563(97.3) & $297(8.1)$ & 3364(91.9) \\
\hline $5-10$ & $11,710(36.2)$ & $405(3.5)$ & $11,305(96.5)$ & 1118(9.5) & $10,592(90.5)$ \\
\hline $11+$ & $16,945(52.4)$ & $706(4.2)$ & $16,239(95.8)$ & $1741(10.3)$ & $15,204(89.7)$ \\
\hline \multicolumn{6}{|l|}{ Specific Medications (ATC code) } \\
\hline Vitamin K antagonists (B01AA) & $4572(14.1)$ & 219(4.8) & 4353(95.2) & $535(11.7)$ & 4037(88.3) \\
\hline SSRIs (N06AB) & $6744(20.9)$ & $307(4.6)$ & $6437(95.4)$ & $743(11.0)$ & $6001(89.0)$ \\
\hline \multicolumn{6}{|l|}{ Activities of Daily Living Level } \\
\hline No or minimal impairment & $12,021(37.2)$ & $604(5.0)$ & $11,417(95.0)$ & 1155(9.6) & $10,866(90.4)$ \\
\hline Mild impairment & $11,242(34.8)$ & $386(3.4)$ & $10,856(96.6)$ & $1231(11.0)$ & $10,011(89.0)$ \\
\hline Moderate impairment & $7182(22.2)$ & $144(2.0)$ & 7038(98.0) & $608(8.5)$ & 6574(91.5) \\
\hline Severe impairment & $426(1.3)$ & $9(2.1)$ & $417(97.9)$ & $24(5.6)$ & 402(94.4) \\
\hline \multicolumn{6}{|l|}{ Behavioural Daily Living Level } \\
\hline No or minimal impairment & $11,506(35.6)$ & $547(4.8)$ & $10,959(95.2)$ & 1314(11.4) & $10,192(88.6)$ \\
\hline Mild impairment & 8682(26.9) & $314(3.6)$ & $8368(96.4)$ & $831(9.6)$ & $7851(90.4)$ \\
\hline Moderate impairment & $7385(22.9)$ & 198(2.7) & 7187(97.3) & $626(8.5)$ & 6759(91.5) \\
\hline Severe impairment & $3298(10.2)$ & $84(2.5)$ & $3214(97.5)$ & $247(7.5)$ & $3051(92.5)$ \\
\hline \multicolumn{6}{|l|}{ Complex Health Care (CHC) Level } \\
\hline No CHC needed & $13,008(40.3)$ & $583(4.5)$ & $12,425(95.5)$ & 1286(9.9) & $11,722(90.1)$ \\
\hline 1-4 CHC procedures needed & $8753(27.1)$ & $316(3.6)$ & $8437(96.4)$ & $944(10.8)$ & 7809(89.2) \\
\hline
\end{tabular}


Table 1 (continued)

\begin{tabular}{|c|c|c|c|c|c|}
\hline Characteristics & Total & Fall within 90 days & No fall within 90 days & Fall within 365 days & No fall within 365 days \\
\hline 5-9 CHC procedures needed & $7286(22.5)$ & 208(2.9) & 7078(97.1) & $660(9.1)$ & 6626(90.9) \\
\hline$\geq 10 \mathrm{CHC}$ procedures needed & $1824(5.6)$ & $36(2.0)$ & 1788(98.0) & $128(7.0)$ & 1696(93.0) \\
\hline \multicolumn{6}{|c|}{ Number Unplanned Hospitalisations (year prior) } \\
\hline None & $12,820(39.7)$ & $367(2.9)$ & $12,453(97.1)$ & 1052(8.2) & $11,768(91.8)$ \\
\hline 1 & $8661(26.8)$ & $333(3.8)$ & 8328(96.2) & $860(9.9)$ & $7801(90.1)$ \\
\hline $2-4$ & $9194(28.5)$ & $419(4.6)$ & $8775(95.4)$ & 1038(11.3) & $8156(88.7)$ \\
\hline $5+$ & $1641(5.1)$ & $90(5.5)$ & $1551(94.5)$ & 206(12.6) & 1435(87.4) \\
\hline \multicolumn{6}{|c|}{ Number of ED Presentations (year prior) } \\
\hline None & $11,690(36.2)$ & $339(2.9)$ & $11,351(97.1)$ & $960(8.2)$ & $10,730(91.8)$ \\
\hline 1 & $9072(28.1)$ & $310(3.4)$ & 8762(96.6) & $837(9.2)$ & 8235(90.8) \\
\hline $2-4$ & $9419(29.1)$ & $453(4.8)$ & 8966(95.2) & $1102(11.7)$ & $8317(88.3)$ \\
\hline $5+$ & $2135(6.6)$ & 107(5.0) & 2028(95.0) & $257(12.0)$ & 1878(88.0) \\
\hline \multicolumn{6}{|c|}{ Number of GP Attendances (year prior) } \\
\hline 0 & $840(2.6)$ & $13(1.5)$ & $827(98.5)$ & $47(5.6)$ & 793(94.4) \\
\hline $1-5$ & $7768(24.0)$ & $247(3.2)$ & $7521(96.8)$ & $712(9.2)$ & 7056(90.8) \\
\hline $6-15$ & $15,698(48.6)$ & $607(3.9)$ & $15,091(96.1)$ & $1541(9.8)$ & $14,157(90.2)$ \\
\hline $16+$ & $8010(24.8)$ & $342(4.3)$ & $7668(95.7)$ & $856(10.7)$ & 7154(89.3) \\
\hline
\end{tabular}

IQR Interquartile range, ARIA Accessibility/Remoteness Index of Australia, ROSA Registry of Senior Australians, ATC Anatomical, Therapeutic and Chemical classification codes, SSRIs Selective serotonin reuptake inhibitors, CHC Complex health care, PRAC Permanent residential aged care, MBS Medicare Benefits Schedule, ED Emergency department, GP General practitioner

${ }^{\text {a }}$ Missing data N(\%):Rx-Risk-V 482(1.5), ROSA Frailty index score 65(0.2)

${ }^{b}$ Conditions were ascertained using the aged care eligibility or entry into care assessments. Dementia was ascertained from the aged care eligibility or entry into care assessments and the dispensing of medications for the treatment of dementia

validation cohort. This additional validation cohort included data from a different Australian state captured in ROSA (New South Wales; NSW). For this validation cohort non-Indigenous individuals $\geq 65$ years old who entered a facility in NSW between $01 / 01 / 2009$ and $31 / 12 / 2016(N=70,462)$ were selected. In this cohort, fall-related hospitalisations were ascertained using the first listed 'external cause' diagnosis in the admitted data collection (covering public and private hospitals) and 'diagnosis code' of the ED datasets using ICD-10-AM codes (Supplementary Table 1).

The externally validated FRAT-up [19] was recreated using the ROSA for this cohort, to compare with our developed fall-related hospitalisations-risk assessment tool's predictive ability. Supplementary Table 7 shows the coding algorithm to recreate the FRAT-up using our available datasets and Supplementary Table 8 includes the model's sHR estimates using this tool. Discrimination was examined using the AUC in two out-of-sample cohorts as described above.

\section{Results}

\section{Cohort description}

Of the 32,316 individuals studied, $64.2 \%(N=20,757)$ were women, the median age was 85 years old (IQR $80-89), 50.5 \%(N=16,334)$ had a diagnosis of dementia, and $52.4 \%(N=16,945)$ were on 11 or more medications. Almost half $(47.8 \%, N=15,452)$ of the cohort had six or more comorbid conditions and $21.5 \%(N=5244)$ had a history of falls. At entry into PRAC, $23.5 \%(N=7608)$ had moderate/high impairments of activities of daily living, $33.1 \%(N=10,683)$ had moderate/high daily behavioural needs and $28.1 \%(N=9110)$ had 5 or more complex health care needs. Sixty percent $(N=19,496)$ had atleast one unplanned hospitalisation, $63.8 \%(N=20,626)$ had at least one ED presentation, and almost all (97.4\%, $N=31,476)$ saw a GP in the year prior to PRAC entry. Table 1 includes individual, system and clinical characteristics, Supplementary Tables 2, 3, 4, 5 and 6 includes all other individual, medication and health care-related factors for the study cohort.

After entry into PRAC, 3.7\% $(n=1209)$ of residents had a fall-related hospitalisation within 90 days and $9.8 \%$ $(n=3156)$ within 365 days (Table 1$)$. The cumulative incidence of mortality was $13.7 \%$ (95\%CI 13.3-14.0\%) within 90 days and $29.2 \%$ (95\% CI 28.7-29.7\%) within 365 days.

\section{Predictors of fall-related hospitalisations within 90 days of PRAC entry}

Twenty variables were contributors to fall-related hospitalisation prediction within 90 days (Table 2). Older individuals $(\mathrm{sHR}=1.02,95 \% \mathrm{CI} 1.02-1.03$, per year of 
Table 2 Predictors of fall-related hospitalisations within 90 days of entry into permanent residential aged care ${ }^{a}$

\begin{tabular}{|c|c|c|c|}
\hline Variables & sHR & $95 \% \mathrm{Cl}$ & $P$-value \\
\hline Time trend, years & 1.06 & $1.03-1.09$ & $<0.001$ \\
\hline Age (per 1 year increment) & 1.02 & $1.02-1.03$ & $<0.001$ \\
\hline Men vs Women & 1.16 & $1.02-1.30$ & 0.028 \\
\hline History of Fractures (yes vs no) & 1.87 & $1.63-2.15$ & $<0.001$ \\
\hline History of Falls (yes vs no) & 1.41 & $1.21-1.64$ & $<0.001$ \\
\hline Dementia (yes vs no) & 1.39 & $1.22-1.57$ & $<0.001$ \\
\hline Delirium (yes vs no) & 1.28 & $1.02-1.61$ & 0.033 \\
\hline \multicolumn{4}{|l|}{ Rx-Risk-V Co-morbidity Category } \\
\hline $2-3$ vs $0-1$ & 1.10 & $0.82-1.46$ & 0.530 \\
\hline $4-5$ vs $0-1$ & 1.29 & $0.97-1.71$ & 0.080 \\
\hline $6-8$ vs $0-1$ & 1.20 & $0.89-1.63$ & 0.240 \\
\hline $9+$ vs $0-1$ & 1.28 & $0.91-1.79$ & 0.160 \\
\hline \multicolumn{4}{|c|}{ Medications Sedative Load Rating Category } \\
\hline $1-2$ vs 0 & 0.93 & $0.75-1.14$ & 0.460 \\
\hline $3+$ vs 0 & 1.11 & $0.95-1.30$ & 0.170 \\
\hline $\begin{array}{l}\text { Vitamin K Antagonists (ATC code } \\
\text { B01AA) (yes vs no) }\end{array}$ & 1.33 & $1.12-1.59$ & 0.001 \\
\hline SSRI (ATC code N06AB) (yes vs no) & 1.21 & $1.03-1.42$ & 0.020 \\
\hline \multicolumn{4}{|l|}{ Activities of Daily Living Level } \\
\hline No or minimal vs Severe impairment & 2.24 & $0.76-6.56$ & 0.140 \\
\hline Mild vs Severe impairment & 1.19 & $0.80-1.76$ & 0.400 \\
\hline Moderate vs Severe impairment & 1.06 & $0.88-1.28$ & 0.520 \\
\hline \multicolumn{4}{|l|}{ Behavioural Daily Living Level } \\
\hline No or minimal vs Severe impairment & 0.91 & $0.66-1.24$ & 0.530 \\
\hline Mild vs Severe impairment & 0.76 & $0.64-0.90$ & 0.002 \\
\hline Moderate vs Severe impairment & 0.88 & $0.74-1.04$ & 0.120 \\
\hline \multicolumn{4}{|l|}{ Mobility Rating } \\
\hline A best vs $D$ worst & 0.46 & $0.24-0.88$ & 0.019 \\
\hline B vs D worst & 0.59 & $0.41-0.86$ & 0.005 \\
\hline C vs D worst & 0.67 & $0.54-0.84$ & 0.001 \\
\hline \multicolumn{4}{|l|}{ Toileting Rating } \\
\hline A best vs $D$ worst & 0.70 & $0.42-1.14$ & 0.150 \\
\hline B vs D worst & 0.67 & $0.47-0.97$ & 0.032 \\
\hline C vs D worst & 0.97 & $0.78-1.19$ & 0.740 \\
\hline \multicolumn{4}{|l|}{ Cognitive Rating } \\
\hline A best vs $D$ worst & 0.59 & $0.47-0.74$ & $<0.001$ \\
\hline B vs D worst & 0.72 & $0.60-0.86$ & $<0.001$ \\
\hline C vs D worst & 0.78 & $0.68-0.89$ & $<0.001$ \\
\hline \multicolumn{4}{|l|}{ Depression and Dysthymia Rating } \\
\hline A best vs $D$ worst & 0.97 & $0.80-1.17$ & 0.760 \\
\hline B vs D worst & 1.01 & $0.82-1.23$ & 0.950 \\
\hline C vs D worst & 1.20 & $0.97-1.49$ & 0.088 \\
\hline \multicolumn{4}{|c|}{ Number of unplanned hospitalisations (year prior) } \\
\hline 1 vs 0 & 1.03 & $0.86-1.25$ & 0.720 \\
\hline $2-4$ vs 0 & 1.04 & $0.86-1.26$ & 0.710 \\
\hline $5+$ vs 0 & 1.27 & $0.97-1.65$ & 0.083 \\
\hline \multicolumn{4}{|l|}{ Number of ED Presentations (year prior) } \\
\hline 1 vs 0 & 1.03 & $0.86-1.24$ & 0.740 \\
\hline $2-4$ vs 0 & 1.36 & $1.11-1.67$ & 0.003 \\
\hline
\end{tabular}

Table 2 (continued)

\begin{tabular}{llll}
\hline Variables & sHR & $\mathbf{9 5 \% C l}$ & $\boldsymbol{P}$-value \\
\hline $\begin{array}{l}\text { 5+ vs } 0 \\
\text { Number of GP Attendances (year prior) }\end{array}$ & 1.21 & $0.90-1.63$ & 0.210 \\
$1-5$ vs 0 & 2.34 & $1.15-4.78$ & 0.019 \\
$6-15$ vs 0 & 2.93 & $1.42-6.03$ & 0.004 \\
$16+$ vs 0 & 3.31 & $1.58-6.93$ & 0.002 \\
\hline
\end{tabular}

sHR Subdistribution hazard ratio, CI Confidence intervals, GP General practitioners, ATC Anatomical, Therapeutic and Chemical classification codes, ED Emergency department, MBS Medicare Benefits Schedule, SSRI Selective serotonin reuptake inhibitor

${ }^{a} N=30,871$ included in models. 1445 (4.5\%) of cohort not included due to missing data

increasing age) and men $(\mathrm{sHR}=1.16,95 \% \mathrm{CI} 1.02-1.30)$ were at a higher risk of fall-related hospitalisations. The strongest predicting factors included history of fractures $(\mathrm{sHR}=1.87,95 \% \mathrm{CI} 1.63-2.15)$, history of falls $(\mathrm{sHR}=1.41,95 \%$ CI 1.21-2.15), dementia $(\mathrm{sHR}=1.39$, $95 \%$ CI $1.22-1.57$ ), and history of delirium $(\mathrm{sHR}=1.28$, 95\%CI 1.02-1.61). In addition, use of a vitamin $\mathrm{K}$ antagonist (which includes the oral anticoagulant warfarin, $\mathrm{sHR}=1.33$, 95\%CI 1.12-1.59) or a selective serotonin reuptake inhibitor (SSRI) antidepressant medication ( $\mathrm{sHR}=1.21,95 \% \mathrm{CI} 1.03-1.42)$ were fall-related hospitalisation predictors. Higher numbers of GP attendances in the year prior to entry into PRAC were associated with a dose-dependent increase in the risk of fall-related hospitalisations, with those who saw a GP $\geq 16$ times, having a 3-times higher risk ( $\mathrm{sHR}=3.31,95 \% \mathrm{CI} 1.58-6.93)$ compared to those who did not see a GP. By comparison to individuals with the worst mobility or impaired cognition ratings, those with less severe or no mobility or impaired cognition, respectively, were dose-dependently less likely to have a fall-related hospitalisation (e.g. individuals in the highest mobility, $\mathrm{sHR}=0.46,95 \% \mathrm{CI} 0.24-0.88$ or cognitive rating category, $\mathrm{sHR}=0.59,95 \% \mathrm{CI} 0.47-0.74$, respectively) (Table 2).

\section{Predictors of fall-related hospitalisations within 365 days of PRAC entry}

Twenty-seven variables were predictors of fall-related hospitalisations within 365 days (Table 3). Older individuals were at a higher risk of fall-related hospitalisations $(\mathrm{sHR}=1.02,95 \% \mathrm{CI} 1.02-1.03$, per year of increasing age) (Table 3 ) and the strongest predictors included dementia ( $\mathrm{sHR}=1.36,95 \% \mathrm{CI} 1.24-1.50)$, history of falls $(\mathrm{sHR}=1.30,95 \%$ CI $1.20-1.42)$, history of fractures $(\mathrm{sHR}=1.28,95 \% \mathrm{CI} 1.15-1.41)$, and osteoporosis $(\mathrm{sHR}=1.13,95 \% \mathrm{CI} 1.03-1.25)$. In terms of medication use, those dispensed a vitamin $\mathrm{K}$ antagonist $(\mathrm{sHR}=1.30,95 \% \mathrm{CI} 1.17-1.45)$ or a SSRI $(\mathrm{sHR}=1.15$, 
Table 3 Predictors of fall-related hospitalisations within 365 days of entry into permanent residential aged care ${ }^{a}$

\begin{tabular}{|c|c|c|c|}
\hline Variables & sHR & $95 \% \mathrm{Cl}$ & $P$-value \\
\hline Time trend, years & 1.07 & $1.05-1.09$ & $<0.001$ \\
\hline Age (per 1 year increment) & 1.02 & $1.02-1.03$ & $<0.001$ \\
\hline Men vs Women & 1.00 & $0.94-1.08$ & 0.900 \\
\hline \multicolumn{4}{|l|}{ Facility Remoteness } \\
\hline Inner Regional vs Major Cities & 0.80 & $0.72-0.91$ & $<0.001$ \\
\hline Outer Regional vs Major Cities & 0.93 & $0.80-1.07$ & 0.300 \\
\hline Remote or Very Remote vs Major Cities & 0.91 & $0.61-1.35$ & 0.640 \\
\hline History of Fractures (yes vs no) & 1.28 & $1.15-1.41$ & $<0.001$ \\
\hline History of Falls (yes vs no) & 1.30 & $1.20-1.42$ & $<0.001$ \\
\hline Dementia (yes vs no) & 1.36 & $1.24-1.50$ & $<0.001$ \\
\hline Osteoporosis (yes vs no) & 1.13 & $1.03-1.25$ & 0.013 \\
\hline \multicolumn{4}{|l|}{ Normalised Weighted ROSA Frailty Index Category } \\
\hline$[0.2-0.4)$ vs $[0-0.2)$ & 0.79 & $0.65-0.96$ & 0.019 \\
\hline$[0.4-0.6)$ vs $[0-0.2)$ & 0.76 & $0.62-0.93$ & 0.007 \\
\hline$[0.6-0.8)$ vs $[0-0.2)$ & 0.71 & $0.57-0.89$ & 0.003 \\
\hline$[0.8-1.0]$ vs $[0-0.2)$ & 0.47 & ND & 0.870 \\
\hline \multicolumn{4}{|l|}{ Rx-Risk-V Co-morbidity Category } \\
\hline $2-3$ vs $0-1$ & 0.99 & $0.84-1.17$ & 0.900 \\
\hline $4-5$ vs $0-1$ & 1.11 & $0.94-1.30$ & 0.210 \\
\hline $6-8$ vs $0-1$ & 1.07 & $0.90-1.28$ & 0.440 \\
\hline $9+$ vs $0-1$ & 1.14 & $0.95-1.37$ & 0.170 \\
\hline Vitamin K Antagonists (ATC Code B01AA) (yes vs no) & 1.30 & $1.17-1.45$ & $<0.001$ \\
\hline SSRI (ATC Code N06AB) (yes vs no) & 1.15 & $1.04-1.27$ & 0.007 \\
\hline \multicolumn{4}{|l|}{ Activities of Daily Living Level } \\
\hline No or minimal vs Severe impairment & 1.01 & $0.56-1.83$ & 0.970 \\
\hline Mild vs Severe impairment & 0.99 & $0.80-1.22$ & 0.930 \\
\hline Moderate vs Severe impairment & 1.02 & $0.88-1.18$ & 0.810 \\
\hline \multicolumn{4}{|l|}{ Behavioural Daily Living Level } \\
\hline No or minimal vs Severe impairment & 0.93 & $0.78-1.11$ & 0.440 \\
\hline Mild vs Severe impairment & 0.89 & $0.78-1.00$ & 0.059 \\
\hline Moderate vs Severe impairment & 0.92 & $0.84-1.01$ & 0.077 \\
\hline \multicolumn{4}{|l|}{ Complex Health Care (CHC) Needs Level } \\
\hline No vs $\geq 10 \mathrm{CHC}$ procedures needed & 1.02 & $0.87-1.20$ & 0.820 \\
\hline $1-4$ vs $\geq 10 \mathrm{CHC}$ procedures needed & 1.07 & $0.97-1.18$ & 0.150 \\
\hline $5-9$ vs $\geq 10 \mathrm{CHC}$ procedures needed & 1.11 & $1.01-1.22$ & 0.026 \\
\hline \multicolumn{4}{|l|}{ Nutrition Rating } \\
\hline A best vs $D$ worst & 1.57 & $1.27-1.94$ & $<0.001$ \\
\hline B vs D worst & 1.46 & $1.22-1.74$ & $<0.001$ \\
\hline C vs D worst & 1.59 & $1.40-1.81$ & $<0.001$ \\
\hline \multicolumn{4}{|l|}{ Mobility Rating } \\
\hline A best vs $D$ worst & 0.74 & $0.53-1.04$ & 0.088 \\
\hline B vs D worst & 0.92 & $0.74-1.14$ & 0.450 \\
\hline C vs D worst & 0.99 & $0.85-1.15$ & 0.880 \\
\hline \multicolumn{4}{|l|}{ Cognitive Rating } \\
\hline A best vs $D$ worst & 0.63 & $0.53-0.75$ & $<0.001$ \\
\hline B vs D worst & 0.78 & $0.69-0.88$ & $<0.001$ \\
\hline C vs D worst & 0.80 & $0.71-0.89$ & $<0.001$ \\
\hline \multicolumn{4}{|l|}{ Wandering Rating } \\
\hline A best vs $D$ worst & 0.88 & $0.78-1.00$ & 0.053 \\
\hline
\end{tabular}


Table 3 (continued)

\begin{tabular}{|c|c|c|c|}
\hline Variables & sHR & $95 \% \mathrm{Cl}$ & $P$-value \\
\hline B vs D worst & 1.09 & $0.93-1.26$ & 0.290 \\
\hline C vs D worst & 1.04 & $0.87-1.23$ & 0.690 \\
\hline \multicolumn{4}{|c|}{ Depression and Dysthymia Rating } \\
\hline A best vs $D$ worst & 0.92 & $0.81-1.05$ & 0.200 \\
\hline B vs D worst & 0.98 & $0.86-1.11$ & 0.710 \\
\hline C vs D worst & 1.07 & $0.94-1.23$ & 0.310 \\
\hline \multicolumn{4}{|c|}{ Number of Unplanned Hospitalisations (year prior) } \\
\hline 1 vs 0 & 1.30 & $1.10-1.52$ & 0.002 \\
\hline $2-4$ vs 0 & 1.45 & $1.21-1.75$ & $<0.001$ \\
\hline $5+$ vs 0 & 1.90 & $1.51-2.39$ & $<0.001$ \\
\hline \multicolumn{4}{|c|}{ Number of ED Presentations (year prior) } \\
\hline 1 vs 0 & 1.07 & $0.95-1.20$ & 0.270 \\
\hline $2-4$ vs 0 & 1.29 & $1.13-1.48$ & $<0.001$ \\
\hline $5+$ vs 0 & 1.28 & $1.06-1.54$ & 0.011 \\
\hline $\begin{array}{l}\text { Number of Unplann } \\
\text { prior) }\end{array}$ & & - & - \\
\hline 1 vs 0 & 0.97 & $0.87-1.09$ & 0.630 \\
\hline $2-4$ vs 0 & 0.85 & $0.72-1.02$ & 0.082 \\
\hline $5+$ vs 0 & 0.23 & $0.02-2.75$ & 0.250 \\
\hline \multicolumn{4}{|c|}{ Days Between Last Unplanned Hospitalisation and Entry into PRAC } \\
\hline $0-20$ vs $151+$ & 0.72 & $0.62-0.84$ & $<0.001$ \\
\hline $21-100$ vs $151+$ & 0.80 & $0.71-0.90$ & $<0.001$ \\
\hline $101-150$ vs $151+$ & 0.75 & $0.63-0.90$ & 0.002 \\
\hline \multicolumn{4}{|c|}{ Number of GP Attendances (year prior) } \\
\hline $1-5$ vs 0 & 1.59 & $1.13-2.22$ & 0.007 \\
\hline $6-15$ vs 0 & 1.75 & $1.25-2.45$ & 0.001 \\
\hline $16+$ vs 0 & 1.99 & $1.41-2.83$ & $<0.001$ \\
\hline \multicolumn{4}{|c|}{ Number of GP Management, Multidisciplinary/Team Care Plans (year prior) } \\
\hline 1 vs 0 & 1.03 & $0.91-1.18$ & 0.610 \\
\hline $2-4$ vs 0 & 1.00 & $0.92-1.09$ & 0.980 \\
\hline $5+$ vs 0 & 1.04 & $0.81-1.33$ & 0.770 \\
\hline \multicolumn{4}{|c|}{ Number of $75+$ Health Assessments (year prior) } \\
\hline 1 vs 0 & 1.08 & $0.98-1.19$ & 0.130 \\
\hline $2+$ vs 0 & 0.99 & $0.69-1.43$ & 0.970 \\
\hline
\end{tabular}

sHR Subdistribution hazard ratio, Cl Confidence intervals, ROSA Registry of Senior Australians, ATC Anatomical, Therapeutic and Chemical classification codes, GP General practitioners, ED Emergency department, MBS Medicare Benefits Schedule, ND Not defined, confidence interval not defined due to inflated standard error

${ }^{a} N=30,808$ included in models. 1508 (4.7\%) of cohort not included due to missing data

95\%CI 1.04-1.27) had a higher risk of having a fallrelated hospitalisation. By comparison to individuals with poor nutrition, those with better nutrition ratings had a greater risk of fall-related hospitalisations (e.g. highest level of nutrition rating $\mathrm{sHR}=1.57,95 \% \mathrm{CI}$ 1.27-1.94). Increasing numbers of unplanned hospitalisations and ED presentations in the year prior to entering PRAC were associated with a higher fall-related hospitalisation risk (e.g. $\geq 5$ unplanned hospitalisations $\mathrm{sHR}=1.90,95 \% \mathrm{CI} 1.51-2.39$ and $\geq 5 \mathrm{ED}$ presentations $\mathrm{sHR}=1.28,95 \% \mathrm{CI} 1.06-1.54)$. Increasing numbers of GP attendances in the year prior to entry were associated with a dose-dependent higher risk of fall-related hospitalisations, with those who had $\geq 16$ GP visits having a 2 -fold ( $\mathrm{sHR}=1.99,95 \% \mathrm{CI} 1.41-2.83$ ) higher risk compared to those who did not see a GP. Compared to individuals with the lowest level of frailty, those with higher frailty scores had lower risks of fall-related hospitalisations (e.g. frailty index $0.6-0.8 \mathrm{sHR}=0.71,95 \% \mathrm{CI} 0.57$ 0.89) (Table 3). Higher cognitive function was associated with a lower risk of fall-related hospitalisation within 365 days (e.g. individuals in the highest cognitive rating 
category sHR $=0.63,95 \% \mathrm{CI} 0.53-0.75)$. The more recent an unplanned hospitalisation to the time of PRAC entry, the less likely individuals were to have a fall-related hospitalisation (e.g. 0-20 days from hospitalisation to entering PRAC $\mathrm{sHR}=0.72$, 95\% CI 0.62-0.84).

\section{Model performance (out of sample and compared to FRAT-up)}

Validation of the risk prediction model using the 20\% testing cohort $(N=6463)$ showed an out-of-sample predictive ability (AUC) of 0.71 (95\%CI $0.68-0.74)$ for fallrelated hospitalisation within 90 days and $0.64(95 \% \mathrm{CI}$ 0.62-0.67) for fall-related hospitalisation within 365 days (Table 4, Fig. 1). This was higher than when compared to the FRAT-up model for both 90 days $(0.66,95 \%$ CI 0.62 $0.69)$ and 365 days $(0.60,95 \%$ CI $0.58-0.66)$. Predictive ability for the model and the FRAT-up model was lower in the NSW out-of-sample validation cohort for 90 days (0.62, 95\% CI 0.61-0.63) and 365 days $(0.60,95 \%$ CI 0.59 0.61) (Table 4, Fig. 1).

\section{Discussion}

A risk prediction model for fall-related hospitalisations for individuals entering PRAC was developed using individual, system, medication, and health care related characteristics routinely collected in Australia. Twenty and 27 factors contributing to the risk of fall-related hospitalisation within 90 days and 365 days of entry into PRAC, respectively were identified. With approximately 70,000 individuals entering PRAC each year and assessment of

Table 4 Proposed prognostic model and FRAT-up model performance to estimate risk of fall-related hospitalisations within 90 and 365 days post entry into permanent residential aged care

\begin{tabular}{lcc}
\hline & \multicolumn{2}{c}{ Model performance, AUC (95\%Cl) } \\
\cline { 2 - 3 } & $\begin{array}{l}\text { Out-of-sample } \\
\text { validation, SA }\end{array}$ & $\begin{array}{l}\text { Out-of-sample } \\
\text { validation, } \\
\text { NSW }^{\mathbf{b}}\end{array}$ \\
\hline Fall-related hospitalisations within 90 days & \\
Proposed Model & $0.71(0.68-0.74)$ & $0.62(0.61-0.63)$ \\
FRAT-up Model & $0.66(0.62-0.69)$ & $0.60(0.59-0.61)$ \\
Fall-related hospitalisations within 365 days & \\
Proposed Model & $0.64(0.62-0.67)$ & $0.60(0.59-0.60)$ \\
FRAT-up Model & $0.60(0.58-0.66)$ & $0.58(0.57-0.58)$ \\
\hline
\end{tabular}

AUC Area under the receiver operating characteritics curve, $\mathrm{Cl}$ Confidence intervals, SA South Australia, NSW New South Wales

${ }^{a}$ Area under the receiver operating characteristic curve

b Validation performed on $20 \%$ South Australian cohort not used in models' development

'Validation performed on individuals' entering residential aged care in New South Wales between 2012 and 2017 clinical needs occurring during this transition period, this is an important time for clinicians and aged care providers to identify, counsel, and minimise the risk of falls and therefore fall-related hospitalisations. The recent Royal Commission into Aged Care Quality and Safety in Australia highlighted the need for a more resident-centred model of care, greater allied health input into resident care, and widespread integration of electronic care systems in facilities, all of which could contribute to mitigating risk of falls [31]. Additionally, a new national quality indicator to monitor the proportion of residents with a fall and a fall that results in a major injury will be incorporated into Australia's National Aged Care Mandatory Quality Indicator Program in July 2021 [32]. Risk prediction tools that can be automated and applied to identify those at risk of falls and prompt intervention at PRAC entry are likely to be valued by aged care providers, clinicians, residents and family members.

This study found that $3.7 \%$ and $9.8 \%$ of residents have at least one fall-related hospitalisation within 90 days and 365 days of PRAC entry, respectively. Importantly, we examined time to fall-related hospitalisation while accounting for the competing risk of mortality [33], and determined the most influential predictors. Our analysis contributes to the existing literature by uniquely determining the predictive ability of models applied to a large cohort of individuals at a specific time period in their aged care journey. Although a significant body of literature surrounds the development of risk profiling tools for falls [13-15, 34], most studies have examined the risk of any fall rather than fall-related hospitalisations, and few have been validated in the PRAC setting. Most existing falls risk profiling tools have achieved low to moderate predictive ability (AUC 0.55-0.65) [14, 34]. Few studies have examined the discrimination ability of more sophisticated analytical approaches for fall risk prediction as utilised in our study. Palumbo et al. [14] used a statistical learning approach for their falls prediction model and found that the simpler FRAT-up tool performed similarly. The discrimination of our 365-day model (0.64) is similar to the predictive ability of prior models, however our 90-day model had better, yet moderate predictive ability (0.71).

Risk factors (i.e. factors associated with the outcome) and/or predictors (i.e. factors that contribute to the prediction of the outcome) of falls among older adults are well documented $[9,10,35]$. In our study, several established factors associated with falls were confirmed, including increasing age, being a man, prior history of falls and fractures, challenges with mobility, and cognitive impairment, including dementia and delirium [9, 10]. We confirmed that use of SSRIs is a fall predictor [9, $10,35,36]$. Other system related factors determined to 

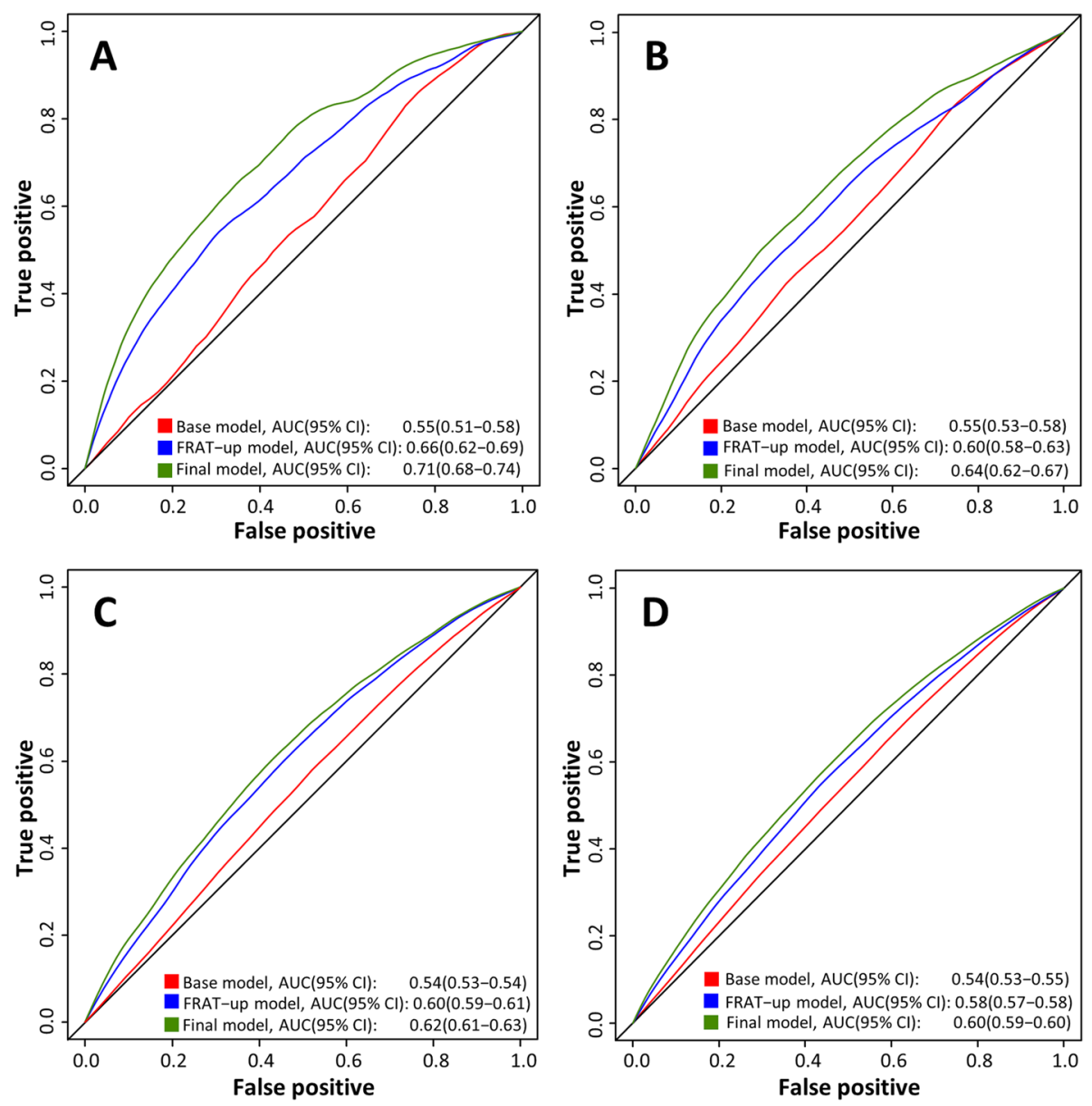

Fig. 1 Proposed prognostic model and FRAT-up model performance to estimate risk of fall-related hospitalisations within 90 and 365 days post entry into permanent residential care out of sample, Area Under the Receiver Operating Characteristics Curve (AUC). A 20\% out-of-sample validation cohort, fall-related hospitalisation within 90 days. B 20\% out-of-sample validation cohort, fall-related hospitalisation within 365 days. C New South Wales validation cohort, fall-related hospitalisation within 90 days. D. South Wales validation cohort, fall-related hospitalisation within 365 days

be fall predictors included the number of GP encounters and unplanned hospitalisations in the year prior to PRAC entry. These have both consistently identified individuals at high risk of hospitalisation [37] and are likely important indications of recent and significant deterioration in individuals' health, and increased need for support.

Use of anticoagulants was found to be associated with a higher risk of fall-related hospitalisations. The association of anticoagulants is likely due to the significant concerns for potentially severe adverse events associated with a fall in an older individual such as increased risk of bleeding [38]. In South Australia, paramedics are trained to transfer all residents taking an anticoagulant to hospital post-fall for further investigation to exclude intracranial injury [39]. We also found the poorest ratings of nutrition and frailty had the lowest risks of fall-related hospitalisations, contrary to other studies $[40,41]$. These paradoxical findings are likely because we accounted for the competing risk of death in our analysis. For example, $67 \%$ of people with the lowest nutrition rating die within a year of PRAC entry compared to only $25 \%$ with higher nutrition ratings. Additionally, those with the worst rating in frailty are likely not ambulant.

Our study strengths include the complete capture of individuals who have entered PRAC in South Australia during the study period. This large sample and linkage of routinely collected aged and health care records allowed us to examine a comprehensive number of predictors systematically. We have also importantly accounted for the competing risk of death in our analysis, which is as high as $35 \%$ in the first year after entering care [42]. The 
validation of our models out-of-sample using 20\% testing cohort in South Australia and from another Australian state, increases the methodological quality of our assessment. Fall-related hospitalisations as the main outcome is an underestimation of the incidence of falls generally, which has been estimated to be as high as $50 \%$ of older people in PRAC facilities [39, 43]. However, it captures significant falls (i.e. resulting in hospitalisation) likely associated with the greatest morbidity, mortality and costs. Our study was also limited to the South Australian cohort, which in 2018 represented $8 \%$ of the new entrants to PRAC nationally [20]. Additionally, only fall-related hospitalisations in public hospitals were included, but this captures $92 \%$ of unplanned hospitalisations in Australia. Our estimate of frailty is also likely underreported as it is captured at the time of the aged care eligibiligy assessment (median time between eligibiligy assessment and entry into PRAC is 258 days) and individuals' frailty levels could have worsened [44]. ROSA does not contain some of the in-depth clinical, environmental, and biological level data that may also contribute to the risk of fall-related hospitalisations at a given point in time. For example, we were unable to examine recent medication dose changes or specific combinations of medication use which may impact fall-related hospitalisation risk. Under-ascertainment of conditions and missing data are possible when using existing datasets. However, these potential limitations were mitigated by conducting logic checks within and between datasets and using multiple points of capture to confirm events/conditions, and the authority responsible for the data linkage in ROSA reports high matching rates (>98\%). Similary, the observational nature of our data means we are unable to infer causality from associations. Finally, predictive ability was lower in the validation cohort, which could reflect state differences in how fall-related hospitalisations are coded or facility protocols related to hospital transfers.

\section{Conclusion}

This exhaustive investigation has identified several individual and health care characteristics, medication, and system-related factors that predict fall-related hospitalisations for older Australians at time of entry into PRAC; a clear point of action. Our findings highlight the ability to utilise integrated routinely collected data to identify residents who are at higher risk of fall-related hospitalisations, thereby providing opportunity to better target strategies to minimise falls risk and related harms.

\section{Abbreviations}

ACAP: Aged Care Assessment Program; ACFI: Aged Care Funding Instrument; AlHW: Australian Institute of Health and Welfare; ATC: Anatomical,
Therapeutical and Chemical; Cl: Confidence intervals; ED: Emergency depart ment; FRAT: Fracture risk assessment tool; GP: General practitioner; IQR: Interquartile range; MBS: Medicare Benefits Schedule; NACDC: National Aged Care Data Clearinghouse; NDI: National Death Index; NSW: New South Wales; PBS: Pharmaceutical Benefits Scheme; PRAC: Permanent Residential Aged Care; ROSA: Registry of Senior Australians; SEIFA: Socio-economic Indexes for Areas; sHR: Sub-distribution hazard ratio; SSRI: Selective serotonin reuptake inhibitor.

\section{Supplementary Information}

The online version contains supplementary material available at https://doi. org/10.1186/s12877-021-02640-w.

Additional file 1: Supplementary Table 1. Hospitalisation coding algorithm to identify fall-related hospitalisations. Supplementary Table 2. Individual characteristics at entry into permanent residential aged care by fall-related hospitalisations within 90 days or 365 days of entry (not included in Table 1). Supplementary Table 3. Individual characteristics at entry into permanent residential aged care from Aged Care Funding Instrument Assessment ${ }^{1}$ by fall-related hospitalisations within 90 days or 365 days of entry (not included in Table 1). Supplementary Table 4. Medication use characteristics within 90 days prior to entry into permanent residential aged care by fall-related hospitalisations within 90 days or 365 days of entry (not included in Table 1). Supplementary Table 5. Hospital related health care utilisation within 1 year prior of entry into permanent residential aged care by individuals' fall-related hospitalisations within 90 days or 365 days of entry (not included in Table 1). Supplementary Table 6. Other health care service utilisation within 1 year prior of entry into permanent residential aged care by individuals' fall-related hospitalisations within 90 days or 365 days of entry (not included in Table 1). Supplementary Table 7. Variables and coding used to recreate risk factors included in the Fracture Risk Assessment Tool for Community Dwelling Older People $(\text { FRAT-up) })^{2}$ and prevalence of risk factor in the study cohort. Supplementary Table 8. Risk Estimates Using the Fracture Risk Assessment Tool for Community Dwelling Older People (FRAT-up) ${ }^{2}$.

\section{Acknowledgements}

We would like to acknowledge the Healthy Ageing Research Consortium Investigator Team and the Registry of Senior Australians'(ROSA) South Australian Health and Medical Research Institute Research Team for ensuring the success of the ROSA and support with this study. We also acknowledge the South Australian Government who provide us with support (2017-2021) through the Department for Innovation and Skills, and the Australian Institute of Health and Welfare (AIHW), SA Health, and the NSW Ministry of Health, for the provision of the raw data used in the ROSA, and the Centre for Health Record Linkage, SA NT DataLink and AIHW for the data linkage.

\section{Informed consent}

This study is a restrospective cohort study of existing data obtained from the Australian Government Department of Health and South Australia and New South Waltes state health authorities and integrated by the Australian Institute of Health and Welfare, Centre for Health Record Linkage, and SA NT DataLink. A waiver of informed consent was granted by the ethics committees who reviewed and approved the study due to the de-identified, existing nature of these data.

\section{Authors' contributions}

$\mathrm{MI}, \mathrm{MM}, \mathrm{CW}$ were involved in the conception and design of the work. MI, CW, $M C, R V$, SW were involved in the acquisition of data. All authors were involved in the analysis and interpretation of the work. MI, MM, and GC were involved in drafting the manuscript and all other authors in the revising it for important intellectual content. All authors approved the version of the manuscript to be published.

\section{Funding}

A/Prof Maria Inacio is supported by The Hospital Research Foundation MidCareer Fellowship (MCF-27-2019) and National Health and Medical Research Council (NHMRC) Investigator Grant (APP119378). JKS is supported by a NHMRC Early Career Fellowship (APP1156439). 


\section{Availability of data and materials}

The data for this study were obtained from the Australian Government Department of Health and South Australia and New South Waltes state health authorities and integrated by the Australian Institute of Health and Welfare, Centre for Health Record Linkage, and SA NT DataLink. These data were made available to the researchers under ethical, governance, and confidentiality agreements that do not allow public sharing.

\section{Declarations}

\section{Ethics approval and consent to participate}

Ethics approvals were received from the University of South Australia (ID:200489), AlHW Ethics Committee (EO2018/1/418), South Australian Department for Health and Wellbeing Ethics Committee (HREC/18/SAH/90), and New South Wales Population \& Health Services Research Ethics Committee (2019/ ETH12028). Our research was performed in accordance with the Declaration of Helsinki.

\section{Consent for publication}

Not applicable.

\section{Competing interests}

The authors declare that they have no competing interests.

\section{Author details}

${ }^{1}$ Registry of Senior Australians, South Australian Health and Medical Research Institute, PO Box 11060, Adelaide, SA 5001, Australia. ${ }^{2}$ UniSA Allied Health and Human Performance, University of South Australia, Adelaide, SA, Australia. ${ }^{3}$ College of Medicine and Public Health, Flinders University, Adelaide, SA, Australia. ${ }^{4}$ Southern Adelaide Local Health Network, SA Health, Adelaide, SA, Australia. ${ }^{5}$ Centre for Medicine Use and Safety, Faculty of Pharmacy and Pharmaceutical Sciences, Monash University, Parkville, VIC, Australia. ${ }^{6}$ UniSA Clinical \& Health Sciences, University of South Australia, Adelaide, SA, Australia. ${ }^{7}$ National Health and Medical Research Council, Centre of Research Excellence Frailty Trans-Disciplinary Research to Achieve Healthy Ageing, Adelaide, SA, Australia. ${ }^{8}$ Adelaide Geriatrics Training and Research with Aged Care Centre (GTRAC), University of Adelaide, Adelaide, SA, Australia. ${ }^{9}$ Aged and Extended Care Services, Central Adelaide Local Health Network, SA Health, Adelaide, SA, Australia. ${ }^{10}$ Adelaide Medical School, University of Adelaide, Adelaide, SA, Australia.

Received: 30 June 2021 Accepted: 10 November 2021 Published online: 07 December 2021

\section{References}

1. Australian Government, Australian Institute of Health and Welfare. Trends in hospitalised injury due to falls in older people, 2007-08 to 2016-17. Injury research and statistics series no. 126. Cat. no. INJCAT 206. https:// www.aihw.gov.au/getmedia/427d3a0d-88c2-45c5-bc23-5e3986375bba/ aihw_injcat_206.pdf.aspx?inline=true. Accessed 31 Aug 2020.

2. World Health Organization. Falls. https://www.who.int/news-room/factsheets/detail/falls. Accessed 31 Aug 2020.

3. James SL, Lucchesi LR, Bisignano C, et al. The global burden of falls: global, regional and national estimates of morbidity and mortality from the global burden of disease study 2017. Inj Prev. 2020;26(Supp 1):i3-i1 1. https://doi.org/10.1136/injuryprev-2019-043286.

4. Australian Institute of Health and Welfare. Australia's health 2018. Australia's health series no. 16. AUS 221. Canberra: AlHW; 2018.

5. Bergen $G$, Stevens MR, Burns ER. Falls and fall injuries among adults aged $\geq 65$ years—United States, 2014. MMWR Morb Mortal Wkly Rep. 2016;65(37):993-8.

6. Hartholt KA, van Beeck EF, Polinder S, et al. Societal consequences of falls in the older population: injuries, healthcare costs, and long-term reduced quality of life. J Trauma. 2011;71(3):748-53.

7. Inacio MC, Amare AT, Whitehead C, et al. Factors associated with accessing aged care services in Australia after approval for services: findings from the historical cohort of the Registry of Senior Australians. Australas J Ageing. 2020. https://doi.org/10.1111/ajag.12760.
8. Australian Government. Australian Institute of Health and Welfare. Injury expenditure in Australia 2015-16. https://www.aihw.gov.au/reports/ health-welfare-expenditure/injury-expenditure-in-australia-2015-16/ contents/injury-spending-by-health-system-area. Accessed 31 Aug 2020.

9. Fernando E, Fraser M, Hendriksen J, Kim CH, Muir-Hunter SW. Risk factors associated with falls in older adults with dementia: a systematic review. Physiother Can. 2017;69(2):161-70. https://doi.org/10.3138/ptc.2016-14.

10. Deandrea S, Bravi F, Turati F, Lucenteforte E, La Vecchia C, Negri E. Risk factors for falls in older people in nursing homes and hospitals. A systematic review and meta-analysis. Arch Gerontol Geriatr. 2013;56(3):407-15. https://doi.org/10.1016/j.archger.2012.12.006.

11. Lalic S, Sluggett JK, Ilomaki J, et al. Polypharmacy and medication regimen complexity as risk factors for hospitalization among residents of long-term care facilities: a prospective cohort study. J Am Med Dir Assoc. 2016;17(11):1067.e1-6. https://doi.org/10.1016/j.jamda.2016.08.019.

12. World Health Organization. Global report on falls prevention in older age. https://www.who.int/publications/i/item/who-global-report-on-fallsprevention-in-older-age?ua=1. Accessed 31 Aug 2020.

13. Nunan S, Brown Wilson C, Henwood T, Parker D. Fall risk assessment tools for use among older adults in long-term care settings: a systematic review of the literature. Australas J Ageing. 2018;37(1):23-33. https://doi. org/10.1111/ajag.12476.

14. Palumbo P, Palmerini L, Bandinelli S, Chiari L. Fall risk assessment tools for elderly living in the community: can we do better? PLoS One. 2015;10(12):e0146247. https://doi.org/10.1371/journal.pone.0146247.

15. Park SH. Tools for assessing fall risk in the elderly: a systematic review and meta-analysis. Aging Clin Exp Res. 2018;30(1):1-16. https://doi.org/10. 1007/s40520-017-0749-0.

16. Welberry HJ, Jorm LR, Schaffer AL, et al. Psychotropic medicine prescribing and polypharmacy for people with dementia entering residential aged care: the influence of changing general practitioners. Med J Aust. 2021;215(3):130-6. https://doi.org/10.5694/mja2.51153.

17. Inacio MC, Lang C, Bray SCE, et al. Health status and healthcare trends of individuals accessing Australian aged care programmes over a decade: the Registry of Senior Australians historical cohort. Intern Med J. 2021;51(5):712-24. https://doi.org/10.1111/imj.14871.

18. Inacio MC, Lang C, Caughey GE, et al. The Registry of Senior Australians outcome monitoring system: quality and safety indicators for residential aged care. Int J Qual Health Care. 2020;32(8):502-10. https://doi.org/10. 1093/intqhc/mzaa078.

19. Palumbo P, Klenk J, Cattelani L, et al. Predictive performance of a fall risk assessment tool for community-dwelling older people (FRAT-up) in 4 European cohorts. J Am Med Dir Assoc. 2016;17(12):1 106-13. https://doi. org/10.1016/j.jamda.2016.07.015.

20. Department of Health. 2017-18 report on the operation of the Aged Care Act 1997. Canberra; 2018. https://www.gen-agedcaredata.gov.au/www_ aihwgen/media/ROACA/2017\%E2\%80\%9318-Report-on-the-Operationof-the-Aged-Care-Act\%E2\%80\%931997.pdf

21. World Health Organization Collaborating Centre for Drug Statistics Methodology. ATC/DDD Index 2018 https://www.whocc.no/atc_ddd_index/. Accessed 30 Nov 2021.

22. Australian Bureau of Statistics. Census of population and housing: socioeconomic indexes for areas (SEIFA), Australia, 2016. https://www.abs.gov. au/AUSSTATS/abs@.nsf/Lookup/2033.0.55.001Main+Features12016? OpenDocument.

23. Moldovan M, Khadka J, Visvanathan R, Wesselingh S, Inacio MC. Using elastic nets to estimate frailty burden from routinely collected national aged care data. J Am Med Inform Assoc. 2020;27(3):419-28. https://doi. org/10.1093/jamia/ocz210.

24. Australian Government, Department of Health and Ageing. Aged Care Funding Instrument. User guide. https://webarchive.nla.gov.au/awa/ 20191107024615/https://agedcare.health.gov.au/funding/aged-caresubsidies-and-supplements/residential-care-subsidy/basic-subsidyamount-aged-care-funding-instrument/aged-care-funding-instrumentacfi-user-guide. Accessed 18 June 2020.

25. Australian Government, Department of Health and Ageing. Aged care assessment program data dictionary. Version 3.0. http://www.sph.uwa. edu.au/_data/assets/pdf_file/0008/2413637/ACAP-Data-DictionaryFinal-V3-0.pdf. Accessed 18 June 2020. 
26. Sloan KL, Sales AE, Liu CF, et al. Construction and characteristics of the RxRisk-V: a VA-adapted pharmacy-based case-mix instrument. Med Care. 2003;41(6):761-74. https://doi.org/10.1097/01.MLR.0000064641.84967.B7.

27. Linjakumpu T, Hartikainen S, Klaukka T, Koponen H, Kivela SL, Isoaho R. A model to classify the sedative load of drugs. Int J Geriatr Psychiatry. 2003;18(6):542-4. https://doi.org/10.1002/gps.846.

28. Hugo Centre for Migration and Population Research. Accessibility/ Remoteness Index of Australia Plus (ARIA+) 2016. The University of Adelaide. https://www.adelaide.edu.au/hugo-centre/services/aria. Accessed 10 Sep 2019.

29. Australian Commission on Safety and Quality in Healthcare. A guide to the potentially preventable hospitalisations indicator in Australia. https:// www.safetyandquality.gov.au/wp-content/uploads/2017/03/A-guide-tothe-potentially-preventable-hospitalisations-indicator-in-Australia.pdf.

30. Heagerty PJ, Lumley T, Pepe MS. Time-dependent ROC curves for censored survival data and a diagnostic marker. Biometrics. 2000;56(2):33744. https://doi.org/10.1111/j.0006-341x.2000.00337.x.

31. Royal Commission into Aged Care Quality and Safety. Final report: care, dignity and respect. https://agedcare.royalcommission.gov.au/sites/ default/files/2021-03/final-report-volume-1_0.pdf. Accessed 2 Aug 2021.

32. Australian Government. Department of Health. National Aged Care Mandatory Quality Indicator Program Manual - 2.0 - Part A (Final version). https://www.health.gov.au/resources/publications/national-aged-caremandatory-quality-indicator-program-manual. Accessed 2 Aug 2021.

33. Pencina MJ, D'Agostino RB Sr. Evaluating discrimination of risk prediction models: the C statistic. JAMA. 2015;314(10):1063-4. https://doi.org/10. 1001/jama.2015.11082.

34. Cattelani L, Palumbo P, Palmerini L, et al. FRAT-up, a Web-based fall-risk assessment tool for elderly people living in the community. J Med Internet Res. 2015;17(2):e41. https://doi.org/10.2196/jmir.4064.

35. Ambrose AF, Paul G, Hausdorff JM. Risk factors for falls among older adults: a review of the literature. Maturitas. 2013;75(1):51-61. https://doi. org/10.1016/j.maturitas.2013.02.009.

36. Seppala LJ, Wermelink A, de Vries $M$, et al. Fall-risk-increasing drugs: a systematic review and meta-analysis: II. Psychotropics. J Am Med Dir Assoc. 2018;19(4):371.e11-7. https://doi.org/10.1016/j.jamda.2017.12.098.

37. Zhou H, Della PR, Roberts P, Goh L, Dhaliwal SS. Utility of models to predict 28-day or 30-day unplanned hospital readmissions: an updated systematic review. BMJ Open. 2016;6(6):e011060. https://doi.org/10.1136/ bmjopen-2016-011060.

38. Bo M, Grisoglio E, Brunetti E, Falcone Y, Marchionni N. Oral anticoagulant therapy for older patients with atrial fibrillation: a review of current evidence. Eur J Intern Med. 2017;41:18-27. https://doi.org/10.1016/j.ejim. 2017.03.012.

39. Sluggett JK, Lalic S, Hosking SM, et al. Root cause analysis of fall-related hospitalisations among residents of aged care services. Aging Clin Exp Res. 2020;32(10):1947-57. https://doi.org/10.1007/s40520-019-01407-z.

40. Cheng $\mathrm{MH}$, Chang SF. Frailty as a risk factor for falls among community dwelling people: evidence from a Meta-analysis. J Nurs Scholarsh. 2017;49(5):529-36. https://doi.org/10.1111/jnu.12322.

41. Trevisan C, Crippa A, Ek S, et al. Nutritional status, body mass index, and the risk of falls in community-dwelling older adults: a systematic review and meta-analysis. J Am Med Dir Assoc. 2019;20(5):569-582.e7. https:// doi.org/10.1016/j.jamda.2018.10.027.

42. Inacio MC, Lang CE, Khadka J, et al. Mortality in the first year of aged care services in Australia. Australas J Ageing. 2020;39(4):e537-44. https://doi. org/10.1111/ajag.12833.

43. Barker AL, Nitz JC, Low Choy NL, Haines T. Measuring fall risk and predicting who will fall: clinimetric properties of four fall risk assessment tools for residential aged care. J Gerontol A Biol Sci Med Sci. 2009;64(8):916-24. https://doi.org/10.1093/gerona/glp041.

44. Thompson MQ, Theou O, Adams RJ, Tucker GR, Visvanathan R. Frailty state transitions and associated factors in south Australian older adults. Geriatr Gerontol Int. 2018;18(11):1549-55. https://doi.org/10.1111/ggi.13522.

\section{Publisher's Note}

Springer Nature remains neutral with regard to jurisdictional claims in published maps and institutional affiliations.
Ready to submit your research? Choose BMC and benefit from:

- fast, convenient online submission

- thorough peer review by experienced researchers in your field

- rapid publication on acceptance

- support for research data, including large and complex data types

- gold Open Access which fosters wider collaboration and increased citations

- maximum visibility for your research: over $100 \mathrm{M}$ website views per year

At BMC, research is always in progress.

Learn more biomedcentral.com/submissions 